News from IUPAC

\section{Research and Training in Medicinal Chemistry in South and Central America and Sub-Saharan Africa}

Professor Antonio Monge Vega, Titular Member of the IUPAC Chemistry and Human Health Division Commission on Training and Development (VII.M.2) and Member of the Working Parties on Medicinal Chemistry Curriculum, Guidelines for Natural Product Collaborations, and Training and Research in Medicinal Chemistry in Developing Countries (Centro de Investigacion en Farmacobiologica Aplicada, Universidad de Navarra, 31080, Pamplona, Spain; email: cifa@unav.es), contributed the following article. This overview represents IUPAC's efforts to develop an awareness of the state of medicinal chemistry in different geographic areas of the world and a proposal to achieve more effective international cooperation.

\section{Introduction}

Improving therapeutic and sanitary conditions in different countries is a noble objective that, at present, receives much attention from diverse organizations and governments throughout the world. However, it is well known that therapeutic needs differ from country to country. For example, in some countries, the principal health problems are linked to cardiovascular diseases, degenerative diseases, and cancer, while in others, infectious diseases are the principal causes of morbidity and mortality. In each of these cases, medicine, as a sanitary tool, is part of the universal heritage, with important implications for sanitary and economic interests. Medicinal chemists are the health professionals charged with the responsibility of synthesizing new compounds for testing as part of the discovery process for new medicines.

IUPAC's Medicinal Chemistry Section decided to gather statistical and anecdotal information about the collaboration and the barriers to progress in drug discovery among countries. We were particularly interested in those countries that are at present unable to contribute a significant part of their resources to research and education in the discovery of new medicinal agents. Our initial actions are intended to determine the current situation; subsequently we will seek channels to facilitate better communication between countries. Here we publish the results of our first study, with the aim of stimulating international contact and collaboration.

This first review considers South and Central America and sub-Saharan Africa. A subsequent study

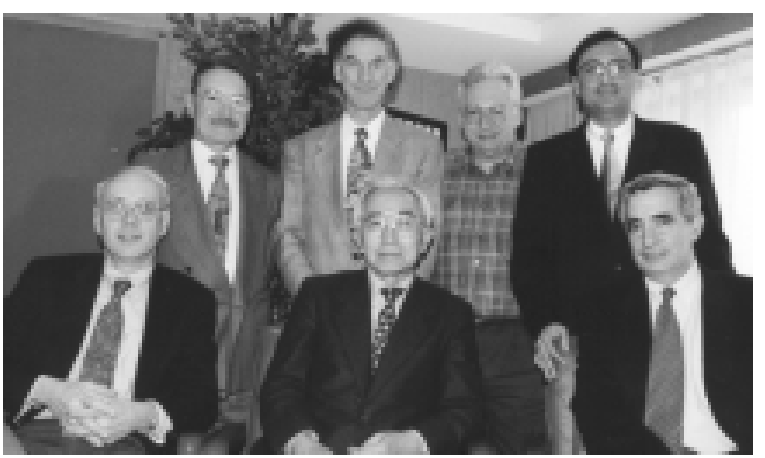

\section{Members of IUPAC Medicinal Chemistry Section}

will include other geographic areas of interest, such as Asia.

\section{Work Rationale}

A medicinal agent is part of the universal heritage of a nation, in spite of the difference between countries that carry out research on new medicinal agents and those countries that are only consumers of medicinal agents. There are countries that promote medicinal chemistry in their universities, research centers, and industrial companies, while in some others, it is common knowledge that this practice is nonexistent. In others still, it is unknown whether this practice is carried out. The world is constantly changing, and great civilizations have disappeared while new ones have emerged. In this context, is the present situation, in which there are countries that carry out research versus countries that only use the results, likely to continue in the future?

The process of discovering medicinal agents contains both old and new elements: the techniques are new, while imagination is old; the instrumentation is new, but careful and well-documented observation is old; and the reporting methods are new and yet communication, as the most characteristic concept for defining humanity, is old. While large research groups usually carry out discovery of medicinal agents, small groups can also be successful when they know their profession well and when their members are researchers of great talents. The discovery and invention of new medicinal agents calls for well-endowed libraries, but access to these installations no longer requires the immediate physical presence of the researcher; such access can be remote. The discovery and invention of new medicinal agents also requires information, but this tool is found not only in traditional libraries. Can we simply ignore old civilizations that have conserved a traditional medicine which has proved itself effective, despite its vicissitudes? 
Events relating to this question date back many years, for example, to the discovery of America in 1492. This example can be applied to today's experience. A continent that was well developed in the arts, philosophy, and sciences, met up with another continent whose development, in general, was very different. These circumstances changed the history of all humanity. But it was in the field of medicine and the area of therapeutic remedies where the great revolution in therapeutics would take place. What has happened since then? The developed countries have considered the traditional medicines of the New World to be of great interest, but they have carried out their research outside the newly discovered territories. This generalization is applicable to Africa, Central America, and South America and has resulted in the development of both research and the derived clinical experience in countries other than those where the native medicinal plants had been found. Thus, many compounds within the scope of modern therapeutics have their origin in plants used in traditional cultures for therapeutic purposes. Current studies with Taxus and Uncaria are examples of this phenomenon.

As a result, the panorama is divided as in the $16^{\text {th }}$ century; however, the situation is not the same. The $21^{\text {st }}$ century will be important for many reasons. The countries of South and Central America and Africa are finding their way in social, political, and even scientific fields. In the near future, true collaboration among countries can be an important alternative, among others, for the global development of medicinal chemistry. Possibly, it is in this context that medicinal chemistry should be developed in the next century.

Cooperation already exists between equals, i.e., welldeveloped companies and well-developed research centers. The time has come for these organizations to cooperate with lesser-developed institutions. Contemplating our planet Earth from up on the Moon clarifies many things with regard to differences between races, countries, cultures, and levels of industrial development.

With the proposal to search for universal cooperation in the field of medicinal chemistry, the IUPAC group has formulated a work plan divided into two phases:

- Develop an awareness of the true situation of medicinal chemistry in the different geographic areas of the world.

- Recommend a proposed set of actions to achieve more effective cooperation.

This report presents and discusses the results of a written questionnaire and interviews carried out in Central and South America and in sub-Saharan Africa.

\section{General Considerations}

The survey was designed to cover four distinct areas:

- teaching of medicinal chemistry
- research in medicinal chemistry

- opportunities for development of research, teaching, and training in the field of medicinal chemistry

- cooperation in practical training, teaching, and research in medicinal chemistry

\section{Survey Recipients}

Addressees of the survey included South and Central American countries and sub-Saharan Africa. Ten surveys were distributed by mail to recipients in Chile (B. K. Cassels and S. Sepúlveda-Boza), Perú (E. Montoya), Nigeria (M. O. Fatope and S. A. Adesanya), Cameroon (N. Barthelemy and B. L. Sondengam), Zimbabwe (L. F. S. Chagonda), Ethiopia (D. Abate), and Madagascar (P. Rasoanaivo).

Surveys were carried out directly by way of interviews in 15 countries of South and Central America. Interviewees included Argentina (G. D. Ferraro), Bolivia (A. G. Turba), Brazil (A. Braga de Oliveira, A. J. Lapa, and E. Barreiro), Colombia (R. Pinzón), Costa Rica (G. A. Mora), Cuba (R. Pellón), Chile (B. Cassels, E. González, and P. Huenchuñir), Ecuador (X. Chiriboga), Guatemala (A. Cáceres), Panamá (M. P. Gupta), Paraguay (E. A. Ferro), Peru (E. Montoya and O. Lock), Dominican Republic (M. Vásquez), Uruguay (E. Manta and G. Seoane), and Venezuela (J. N. Domínguez).

\section{Results and Analysis of the Answers Received}

Results and analysis are grouped according to the four areas described under "General Considerations" above.

\section{Teaching of Medicinal Chemistry}

In this section of the questionnaire, a request was made for information concerning the type of institution where medicinal chemistry is taught, the strong points and deficiencies observed in the system, and the perceived needs and actual situation concerning collaboration with other countries.

The fundamental situation is that teaching of medicinal chemistry, as currently defined, is not present at all in the developing countries; however, complementary disciplines such as organic chemistry, pharmacognosy, and pharmacology are considered. In some cases, studies in medicinal chemistry are related to the identification and preparation of biologically active chemical entities, not new chemical entities.

Results of the surveys indicate that studies related to medicinal chemistry are dispersed throughout different institutions and reflect interest in considering medicinal chemistry as a new discipline to be included in the curriculum.

Interesting initiatives have been found in the promotion of medicinal chemistry education. One noteworthy example is that of a group of professors from 
Brazil (56 Schools of Pharmacy) who are working on new study programs, both academic and training, incorporating internationally recognized tools. This type of initiative is not unique in South America, and a Peruvian group can be cited as one more example. Argentina is yet another interesting case where studies in medicinal chemistry are adapted to current parameters used by those countries with a long tradition in this specialty.

From a teaching standpoint, the needs are, in general, substantial and important. Three areas require scrutiny: (1) teaching staff, (2) reference literature, and (3) reagents and equipment. Teaching staff, in general, are well trained and quite interested in the subject. Very often, many of these professors have had long stays in prestigious universities and research centers as part of their training. Making adjustments within the field of education to allow professors from related disciplines to contribute to the task of teaching medicinal chemistry does not appear to present special difficulties. From a reference literature standpoint, important needs have been found. Lack of a research tradition, vital for consolidating libraries of reference materials, and economic problems in coping with the cost of these essential tools have contributed to important deficiencies in the bibliographic area. Lack of adequate reagents is equally important. Once again, the economic aspects conflict with the teaching practice.

Aid is needed in all three areas of teaching. Experts are needed to give courses in the various institutions surveyed. These experts need to be able to collaborate in drafting training programs and to be able to receive professors for training in their universities. Material aid for acquisition of reference literature and of supplies for practical training in laboratories is a foremost necessity. In addition, inadequate maintenance of laboratory equipment by supplier companies is a frequent problem.

\section{Research in Medicinal Chemistry}

This section of the questionnaire investigated the offerings and needs of research centers and personnel dedicated to the study of medicinal chemistry.

Research in medicinal chemistry in the countries surveyed is and has historically been centered almost exclusively on the field of natural products. This situation can be traced to considerable historical knowledge of plants with medicinal activity in cultures whose origins are very remote, and to the circumstance that little effort has been put into technology and scientific investigation in the search for and improvement of active compounds by molecular manipulation.

All those who answered the questionnaire showed great interest in not being mere suppliers of plants. In order to upgrade medicinal chemistry research in developing countries by taking natural products as a starting point, it is necessary to consider the following:
- Strengths: correct identification of plants (in general, good botanists exist in these countries), gathering of plant material, and preparation of extracts.

- Weaknesses: difficulties in determination of biological activities, validation of extracts, determination of structures responsible for specific biological activity, and industrialization and commercialization.

Independent of the degree of scientific development, there is considerable interest in entering the field of medicinal chemistry research. Scientific authorities in the countries surveyed are particularly interested in collaborating on research relevant to natural products with biological activity. Most importantly, authorities of the countries studied consider the active plants to be part of their heritage and a possible source of wealth. They are aware of the fact that they are obliged to attempt, by all means, to retain the greater part of the resulting capital gains. In some cases, bureaucratic requirements necessary for obtaining plants or extracts from their place of origin are overwhelming, and failure to satisfy these requirements can result in judicial action.

Opportunities for Development of Research, Teaching, and Training in Medicinal Chemistry Although natural products are of greatest interest to the countries surveyed, possibilities for collaboration with countries that are leading the way in research and education in medicinal chemistry are so diverse that they could cover practically any area of interest in the therapeutic field.

In a true sense of collaboration, it is important to work on questions that are characteristic of the countries surveyed. A representative example is Chagas' disease, or malaria. There are also diseases, such as AIDS, that were once restricted to certain countries but are now a universal problem. An illness such as diarrhea, no longer considered to be a significant problem in developed countries, is of great concern in the countries surveyed. Ophthalmologic diseases are another example of interest to developing societies, where there is a different focus from that in developed countries. The approach to treating these diseases can be considered, at least partially, from a medicinal chemistry standpoint.

All of the aforementioned examples are of interest to countries that are developing medicinal chemistry programs. The point here is that working collaboratively on these issues is not only resulting in a great contribution to development of and interaction with other societies, but it is also leading to the discovery of new opportunities for those societies that have found their way into the field of medicinal chemistry. 


\section{Cooperation in Practical Training, Teaching, and Research in Medicinal Chemistry}

The need for increased cooperation appears in all of the questionnaire responses. The impression created in this regard is that there are countries that circulate on a railway on board a train while others run alongside asking for help to get on. The countries on board the train must extend a hand to the countries that are not on board by responding to their requests.

Requests are very diverse. Countries such as Cameroon are considering aid, including in its initial stages, for commercializing plants in which active compounds appear. In general, cooperation is requested for correct preparation of extracts. In other cases, requests are related to the identification of active molecules and, in still others, to the determination of biological activities. Implicit in all the responses is a desire to be able to participate in the processes of research and development so as not to remain mere suppliers of plants.

Cooperation can be set up along three fundamental lines of action:

- courses, seminars, etc., that foster relationships between institutions and persons within the teaching field

- joint scientific investigations and research projects, emphasizing activities that allow for development of research centers

- greater presence of pharmaceutical companies in scientific collaboration with those countries that sell their products

\section{Comments}

Survey responses have not been numerous enough to allow us to propose a statistical study of much significance. Nevertheless, certain tendencies constantly appear, which allow us to come to conclusions that seem to be generally valid.

First of all, similar problems exist in all three areas studied. Activity in medicinal chemistry is linked directly to the degree of progressiveness and economic development of each country. In more than one case, medicinal chemistry has been considered a luxury permitted only in developed countries.

Medicaments have historically been studied by using plants and traditional medicine as a starting point even in the most sophisticated societies. From a pragmatic point of view, it would be interesting to take into account the realities in developing countries when setting up corresponding academic programs and when putting forth proposals for collaboration on scientific research. Scientific authorities in Latin American countries seem to agree that this approach is wise.

With regard to collaboration on aspects of teaching, no special difficulties seem to appear; most likely, this interaction should establish itself based on mutual knowledge and aid from various institutions and foundations. Necessary actions to be taken in this area are not especially difficult; they fundamentally require the generosity of professors and researchers in the dedication of their time. One aspect to take into consideration would be the sometimes difficult access to money for travel expenses for professors.

Special importance should be placed on circulation of reference literature and bibliographic material. Any collaboration in this area should take into account all aspects of intellectual property, including legal rights of publishers, editors, and authors. Finding formulas that allow access to reference information by countries of limited resources is a true challenge to the imagination of the scientific community. Likewise, obtaining the means for developing countries to purchase adequate materials and reagents can only be accomplished through economic collaboration.

Equipment maintenance is an interesting consideration deduced from the survey. Some research centers have had access to valuable equipment as a result of goverment support, aid from private foundations, etc. However, in most of these cases, upkeep of this equipment, which is usually quite costly in monetary terms, has not been taken into consideration. This point is especially important in those countries that cannot readily maintain instruments in working order because maintenance service is not justified on the part of companies that produce the equipment.

With regard to possibilities of collaboration on scientific research, it is necessary to start from a position that contemplates the true situation of the indigenous institutions, including their strengths as well as their weaknesses. At present, it is possible to obtain quality extracts from plants that have proved active in traditional medicine. This advantage is important to those countries that have the plants and know their scientific possibilities; however, the possibility of obtaining extracts from plants that have never been studied before should not be underestimated. Structural novelty now appears on the scene frequently, and with it there is potentially an enormous interest when considering the possibilities of patents. Determination of biological activities, their validation, and elucidation of structures responsible for these activities should all be part of any offer of collaboration from even the most advanced countries. Most importantly, discovery of new medicinal agents cannot be realized without active participation of pharmaceutical companies.

\section{A Crucial Time for Collaboration in Medicinal Chemistry}

We have reached an important juncture for both the developing and advanced countries regarding collabo- 
ration in the field of medicinal chemistry. We need to question whether true cooperation is developing between the groups or if, on the other hand, differences are increasing. We must also question whether we are capable of recognizing the possibilities of collaboration, for mutual benefit, on areas in which the world of research is truly divided at this time.

Our survey shows that the developing countries are requesting collaboration with more advanced societies in which natural products should be considered as a starting point; this opportunity could very well be of interest to both societies.

This report was prepared for publication by Prof. A. Monge Vega (chairman, Spain), Universidad de Navarra, CIFA, Irunlarrea, s/n, 31008 Pamplona, Spain. The IUPAC Committee includes Prof. C. R. Ganellin, Department of Chemistry, Christopher Ingold Laboratories, University College London, 20 Gordon Street, London, WC1H 0AJ, UK; Dr. J. Ide, Medicinal Chemistry Research Laboratories, Sankyo Co., Ltd., 2-58 Hiromachi 1-Chome, Shinagawa-Ku, Tokyo 140, Japan; Dr. N. Koga, Daiichi Pharmaceutical Co. Ltd., 1410, Nihonbashi 3-Chome, Chuo-Ku, Tokyo, 103 Japan; Dr. P. Lindberg, Astra Hassle, A.B., S-432 83, Molndal, Sweden; Prof. L. A. Mitscher, Department of Medicinal Chemistry, School of Pharmacy, University of Kansas, Lawrence, KS 66045-2506, USA; Dr. J. C. Muller, Synthelabo Recherche, BP 110, 31 Avenue Paul Vaillant-Couturier, F 92225 Bagneux Cédex, France; Dr. T. J. Perun, Abbott Laboratories, Dept. 467, Building R-8, 1401 Sheridan Road, North Chicago, IL 600644000, US.A; Prof. J. G. Topliss, University of Michigan, College of Pharmacy, 428 Church St., Department of Medicinal Chemistry, Ann Arbor, MI 48109-1065, USA; Prof. C. G. Wermuth, Faculte de Pharmacie, Laboratoire de Chimie Organique, Universite Louis Pasteur, 74, Route du Rhin, BP 24 Strasbourg, F 67401 Illkirch Cedex, France.

\section{Agenda for $40^{\text {th }}$ IUPAC Council Meeting, Berlin, Germany, 13-14 August 1999}

1. Introductory Remarks and Finalization of Agenda

2. Approval of Minutes of $39^{\text {th }}$ Council Meeting and Matters Arising

3. Ratification of Decisions Taken by Bureau and Executive Committee since $39^{\text {th }}$ General Assembly

4. Announcement of Nominations for Union Officers and Bureau Members

5. Announcement of Time of Elections

6. Statutory Report of President on State of Union

7. Report of Secretary General

8. Biennial Report of Treasurer/Report of Finance Committee and Accounts for 1997-8/Appointment of Auditors for 1999-2000

9. Reports of Division Presidents (10 minutes each)
10. Report of Committee on Printed and Electronic Publications (10 minutes)

11. Report of CHEMRAWN Committee (10 minutes)

12. Report of Committee on Chemistry and Industry (10 minutes)

13. Report of Committee on Teaching of Chemistry (10 minutes)

14. Report on and Review of Affiliate Membership and Fellows Programs

15. Budget Proposal and National Subscriptions for 2000-2001

16. Proposed Changes to Statutes and Bylaws

17. Continuation/Dissolution of Existing IUPAC Bodies, Proposals for New and Reconstituted Bodies/ Terms of Reference

18. Proposals Formally Received from National Adhering Organizations

19. Proposed Category of "Associated National Adhering Organizations"

20. Approval of Dates and Sites of $41^{\text {st }}$ General Assembly and $38^{\text {th }}$ Congress (2001)

21. Approval of Dates and Sites of $42^{\text {nd }}$ General Assembly and $39^{\text {th }}$ Congress (2003)

22. Election of Union Officers and Bureau Members and Approval of Elected Officers of Divisions

23. Applications for Membership in IUPAC

24. Applications for Associated Organization Status within IUPAC

25. Adoption of Recommendations on Nomenclature and Symbols

26. Important Matters Discussed by Bureau at $40^{\text {th }}$ General Assembly not Covered by Items on Council Agenda

27. Any Other Business (discussion only)

\section{Text of Proposed Bylaw Change, Agenda Item 16}

Current

B4.307 Titular Members of Commissions have the right to receive contributions towards travel and subsistence expenses from funds of the Union as authorized by the Treasurer acting on behalf of the Union. Contributions may be made to Associate Members or members of subcommittees on recommendation of the Division or Section President and with the agreement of the Treasurer.

\section{Proposed}

B4.307 Members of IUPAC bodies may receive contributions towards travel and subsistence expenses from funds of the Union, as authorized by the Treasurer. The Bureau shall establish procedures and guidelines for the approval of such expenses. 